\title{
Rinite alérgica - Classificação, fisiopatologia, diagnóstico e tratamento
}

\section{Allergic rhinitis - Classification, pathophysiology, diagnosis and treatment}

Rev Port Imunoalergologia 2021;29 (2):95-106

Leonor Esteves Caldeiral,* (iD, Maria Inês T. Silval,* (D), Gonçalo Martins-dos-Santos² (iD,

Ana Margarida Pereira ${ }^{3,4,5}$ (iD

I Serviço de Imunoalergologia, Hospital Santa Maria, Centro Hospitalar Universitário Lisboa Norte EPE, Lisboa

${ }^{2}$ Serviço de Imunoalergologia, Centro Hospitalar Universitário de Lisboa Central, Lisboa

${ }^{3}$ CUF-Porto Instituto e Hospital, Porto

${ }^{4}$ MEDCIDS - Departamento Medicina da Comunidade, Informação e Decisão em Saúde, Faculdade de Medicina da Universidade do Porto

${ }^{5}$ CINTESIS - Center for Health Technology and Services Research, Faculdade de Medicina da Universidade do Porto

* Ambos os autores contribuíram de forma igual para a escrita deste trabalho

\section{RESUMO}

A rinite alérgica é uma patologia inflamatória da mucosa nasal, mediada por imunoglobulina $\mathrm{E}$ (IgE), que se manifesta por rinorreia mucosa, obstrução nasal, crises esternutatórias e/ou prurido nasal em relação com a exposição a aeroalergénios. A sua classificação depende da duração e gravidade dos sintomas que, quando não controlados, podem apresentar um grande impacto na qualidade de vida. Embora de diagnóstico essencialmente clínico, o recurso a testes cutâneos por picada e doseamento sérico de lgE específicas é importante na confirmação da etiologia e na caraterização dos alergénios envolvidos. $O$ tratamento baseia-se em medidas de controlo ambiental, em terapêutica farmacológica e, em casos selecionados, no uso da imunoterapia com alergénios. Este artigo tem como objetivo reunir a atual evidência científica relativa à rinite alérgica, no que se refere à sua fisiopatologia, diagnóstico e adequada abordagem terapêutica, usando um caso clínico para contextualização do problema.

Palavras-chave: Hipersensibilidade imediata, imunoterapia com alergénios, medicina de precisão, rinite alérgica. 


\section{ABSTRACT}

Allergic rhinitis is an immunoglobulin E (IgE)-mediated inflammatory pathology of the nasal mucosa clinically characterized by mucous rhinorrhea, nasal congestion, sneezing and/or nasal itch. Its classification depends on the duration and severity of symptoms which, when not controlled, can have a major impact on quality of life. Although the diagnosis is mainly based on clinical features, the use of skin prick testing and allergen-specific serum lgE measurements are important to confirm the etiology and characterize the involved allergens. Treatment includes environmental control measures, pharmacological treatment and, in selected cases, allergen immunotherapy. The aim of this article is to gather current scientific evidence on allergic rhinitis, in what concerns its pathophysiology, diagnosis and appropriate management, using a clinical vignette as a starting point.

Keywords: Allergic rhinitis, immediate hypersensitivity, allergen immunotherapy, precision medicine.

(C) 202I Sociedade Portuguesa de Alergologia e Imunologia Clínica. Published by Publicações Ciência \& Vida. This is an open access article under the CC BY-NC-ND license (http://creativecommons.org/licenses/by-nc-nd/4.0/).

N esta série de artigos educacionais sobre rinite optamos pelo formato de iniciar com um caso clínico sumário ilustrando um problema clínico frequente. Posteriormente, é apresentada evidência relativa a essa patologia, focando diferentes aspetos que se consideram clinicamente relevantes, incluindo formas de abordagem. $\mathrm{O}$ artigo termina com as recomendações clínicas dos autores, tendo por base o caso clínico inicial.

\section{CASO CLÍNICO}

Homem, 23 anos, sem antecedentes pessoais relevantes. Referenciado à consulta de Imunoalergologia aos 18 anos por queixas de rinorreia anterior mucosa, obstrução nasal, crises esternutatórias, prurido nasal e ocular e hiperemia conjuntival. Referia agravamento da sintomatologia descrita na primavera e outono, apesar de ter queixas perenes e quase diárias, com interferência nas atividades do dia-a-dia e no sono. Após investigação etiológica estabeleceu-se o diagnóstico de rinoconjuntivite alérgica persistente, moderada-grave, com sensibilização a Dermatophagoides pteronyssinus, Dermatophagoides fari- nae, pólen de gramíneas, de oliveira e de salsola. A espirometria com prova de broncodilatação não mostrou alterações funcionais respiratórias. Foi medicado com furoato de mometasona nasal $50 \mathrm{mcg} /$ dose, 2 pulverizações em cada narina de manhã e à noite, bilastina oral 20 $\mathrm{mg}$ id e cetotifeno ocular $0,25 \mathrm{mg} / \mathrm{ml}$, I gota em cada olho de manhã e à noite, apenas com resposta parcial. Não foi possível suspender a medicação em nenhuma altura do ano por recidiva da sintomatologia descrita.

\section{REVISÃO TEÓRICA}

\section{Introdução}

A rinite alérgica (RA) é uma doença com manifestação predominantemente nasal, determinada por um processo inflamatório mediado por imunoglobulina $\mathrm{E}(\mathrm{lg} \mathrm{E})$ após a exposição da mucosa nasal a um ou mais alergénios'.

Trata-se de uma doença com crescente prevalência, estimando-se atualmente uma prevalência global de 10 a $40 \%$, variando consoante a localização geográfica ${ }^{2}$. Apesar da sua elevada prevalência, a RA é ainda uma doença subdiagnosticada ${ }^{3,4,5}$ e subtratada ${ }^{6,7}$. 
As principais manifestações clínicas da RA são: rinorreia mucosa, obstrução nasal, crises esternutatórias e prurido nasal. Estes sintomas são frequentemente considerados "normais" e consequentemente grande parte dos doentes não reconhecem os seus sintomas como doença e não procuram avaliação médica ${ }^{7}$; adicionalmente, os próprios médicos subestimam frequentemente a gravidade da $R A^{8}$. No entanto, os sintomas nasais, quando presentes, podem ter grande impacto na qualidade de vida, com perturbação das atividades diárias, associada a fadiga e diminuição da energia, bem como a interferência no sono e redução da capacidade de concentração9,10,11. Adicionalmente, a RA está associada a alteração da produtividade laboral e menor aproveitamento escolar ${ }^{12,13,14}$, sendo responsável por custos elevados, especialmente indiretos (por absentismo ou presenteísmo) 15,16,17.

\section{Classificação}

A RA pode ser classificada como sazonal ou perene segundo as características temporais da doença e a exposição ao alergénio'. Contudo, esta classificação não é globalmente aplicável e, consequentemente, o grupo de trabalho internacional Allergic Rhinitis and its Impact on Asthma (ARIA) reformulou as diretrizes da rinite alérgica tendo por base a duração (intermitente, persistente) e gravidade (ligeira, moderada-grave) dos sintomas' (Figura I).
Ambas as abordagens são úteis e não mutuamente exclusivas. A classificação definida pelo ARIA é a mais aceite atualmente e é particularmente útil em países onde a polinose ocorre durante todo o ano, sem estação definida ou muito prolongada. A subdivisão sazonal/perene pode ser mais relevante em países onde há exposições sazonais claras a alergénios ${ }^{18}$.

\section{Fisiopatologia}

A fisiopatologia da RA é complexa, compreendendo uma resposta alérgica de fase precoce e tardia ${ }^{19,20}$. $O$ processo é desencadeado pela exposição a alergénios como pólenes, ácaros, epitélio de animais, entre outros, que são reconhecidos por recetores de IgE específicos existentes na superfície de mastócitos e basófilos de indivíduos pré-sensibilizados. Esta fase tem início minutos após a exposição ao alergénio, dura cerca de 2 a 3 horas e é predominantemente caracterizada pela desgranulação de mastócitos ${ }^{21}$. Os mastócitos são abundantes no compartimento epitelial da mucosa nasal de indivíduos sensibilizados, os quais podem ser facilmente ativados após a reexposição aos alergénios implicados ${ }^{21}$. Após interação do alergénio específico com a lgE ligada à superfície dos mastócitos ocorre a sua desgranulação e a consequente libertação de mediadores pró-inflamatórios, sendo a histamina o principal destes mediadores. A histamina e os outros mediadores, como os leucotrienos e as prosta-

\begin{tabular}{|c|c|}
\hline \multicolumn{2}{|c|}{ Duração dos sintomas } \\
\hline Intermitente & Persistente \\
\hline sintomas $<4$ dias/semana ou $<4$ semanas & sintomas $\geq 4$ dias/semana e $\geq 4$ semanas \\
\hline \multicolumn{2}{|c|}{ Gravidade dos sintomas } \\
\hline $\begin{array}{l}\text { Ligeiras } \\
\text { (todos presentes) }\end{array}$ & $\begin{array}{c}\text { Moderada-grave } \\
\text { (pelo menos I presente) }\end{array}$ \\
\hline $\begin{array}{l}\text { - sem interferência no sono } \\
\text { - sem interferência nas atividades diárias, desportivas ou de lazer } \\
\text { - sem interferências nas atividades da escola ou trabalho } \\
\text { - sem sintomas incomodativos }\end{array}$ & $\begin{array}{l}\text { - perturbação do sono } \\
\text { - perturbação nas atividades diárias, desportivas ou de lazer } \\
\text { - dificuldades nas atividades da escola e/ou trabalho } \\
\text { - sintomas incomodativos }\end{array}$ \\
\hline
\end{tabular}

Figura I. Classificação da rinite alérgica (Adaptado de ARIA'). 
glandinas, atuam nos vasos sanguíneos, terminações nervosas sensoriais e glândulas mucosas causando congestão nasal, prurido e rinorreia ${ }^{21,22}$.

A fase tardia inicia-se cerca de 4 a 8 horas após exposição a um alergénio relevante ${ }^{21}$. É de natureza predominantemente inflamatória e definida pelo recrutamento celular de basófilos, neutrófilos, linfócitos Th2, monócitos e eosinófilos e pela libertação de múltiplos mediadores, incluindo citocinas, prostaglandinas e leucotrienos, que perpetuam a resposta inflamatória. Esta fase está associada ao remodeling e aumento do edema dos tecidos, o que leva ao desenvolvimento e manutenção da congestão nasal, um dos sintomas da RA que provoca maior incómodo ${ }^{23}$. Considera-se que estas alterações de fase tardia contribuem ainda para a hiperreatividade brônquica ${ }^{21}$.

\section{Manifestações clínicas}

A história clínica é fundamental para o diagnóstico preciso da doença, para avaliação da sua gravidade e da resposta à terapêutica'. Os sintomas clássicos da rinite alérgica são prurido nasal, esternutos, rinorreia aquosa e congestão nasal. Frequentemente está também associada a sintomas oculares (prurido ocular e epífora), rinorreia posterior, tosse crónica não produtiva e fadiga ${ }^{24-26}$.

\section{Diagnóstico}

O diagnóstico da RA baseia-se numa história clínica detalhada, exame objetivo e exames complementares de diagnóstico (Quadro I), nomeadamente testes cutâneos por picada (TCP) e/ou doseamento sérico de IgE específicas (slgE) $)^{20,24,27}$. Quando dois ou mais sintomas, entre rinorreia aquosa, obstrução nasal, esternutos e/ou prurido nasal, persistem mais de uma hora na maioria dos dias, e que pioram com exposição a alergénios identificados, perenes ou sazonais, é mais provável a presença de RA ${ }^{20}$.

Na realização do exame objetivo a primeira abordagem é a realização de uma rinoscopia anterior, que é uma técnica simples, facilmente executada, sem necessidade de equipamentos dispendiosos e que permite a visualização do terço anterior da cavidade nasal e também identificar complicações ou outras condições nasais (pólipos nasais, desvio septal). Embora não estejam definidos sinais específicos na rinoscopia, na RA a mucosa encontra-se frequentemente alterada, com uma tonalidade azulada/pálida, podendo também apresentar-se edemaciada e hiperemiada nos estados inflamatórios de agudização ${ }^{28}$. A desvantagem da rinoscopia anterior é o facto de não permitir visualizar toda a cavidade nasal, nomeadamente o meato médio, para onde drenam os seios maxilar, frontal e etmoidal anterior, que tão comummente estão envolvidos na RA. Tal facto pode ser ultrapassado através da realização de uma endoscopia nasal, que permite distinguir de forma mais clara entre patologia estrutural ou da mucosa, ficando no entanto reservada para os doentes que não respondem

Quadro I. Resumo dos métodos de diagnóstico na rinite alérgical,30

\begin{tabular}{|l|l|l|}
\hline \multicolumn{1}{|c|}{ Métodos standard } & \multicolumn{1}{|c|}{ Métodos adicionais } & \multicolumn{1}{c|}{ Métodos de investigação } \\
\hline - História clínica & - Medição de NO nasal & - Genómica \\
- Exame objetivo & - Rinomanometria & - Transcriptómica \\
- Testes cutâneos por picada & - Rinometria acústica & - Microbioma, viroma \\
- Medição sérica de slgE & - Provocação nasal & - Epigenética \\
& - Provocação conjuntival & - Proteómica \\
& & - Lipidómica \\
& & - Metabolómica \\
& & - Cultura celular \\
& & - Biologia molecular \\
\hline
\end{tabular}

slgE - Imunoglobulina E específica; NO - óxido nítrico 
ao tratamento inicial ou que não apresentam alterações na rinoscopia. É também necessário fazer observação da orofaringe para excluir presença de rinorreia posterior. A auscultação pulmonar é importante para avaliar eventual envolvimento brônquico ${ }^{1,27}$.

Relativamente aos meios complementares de diagnóstico, são frequentemente utilizados os TCP com extratos comerciais de aeroalergénios; a medição dos níveis séricos de slgE e/ou as lgE moleculares ( $\mathrm{mlgE}$ ) são especialmente úteis em caso de dúvida diagnóstica - por exemplo, quando o resultado dos TCP é discrepante com a sintomatologia apresentada pelo doente ou quando há polissensibilização (como o doente deste caso). A avaliação do perfil de sensibilização molecular é relevante para um diagnóstico mais preciso e pode ser fundamental na seleção da constituição da imunoterapia com alergénios, a administrar nos doentes com indicação para esse tratamento ${ }^{29}$. De facto, o diagnóstico molecular vem minimizar alguns dos problemas associados ao uso dos extratos alergénicos tradicionais. Estes extratos são compostos sobretudo pelas proteínas dos alergénios (major e minor), podendo também conter outras pequenas moléculas, como glicoproteínas e polissacarídeos ${ }^{30}$. No entanto, a sua estandardização é difícil e a sua composição pode ser muito diferente de acordo com o laboratório em que são produzidos, com alguns componentes alergénicos pouco representados ou ausentes em alguns dos extratos ${ }^{29}$. Esta variabilidade dos extratos pode levar a diferenças significativas nos resultados (positivo vs negativo) e influenciar o diagnóstico ${ }^{29,31}$. O uso de alergénios moleculares, para além de ultrapassar essa incerteza associada à composição dos extratos tradicionais, permite uma melhor avaliação do perfil de sensibilização, distinguindo sensibilizações primárias (específicas de espécie) de reatividade cruzada entre múltiplos alergénios ${ }^{31}$. Esta distinção é particularmente relevante na seleção da composição para imunoterapia com alergénios, especialmente em doentes polissensibilizados ${ }^{31,32}$. No entanto, ainda não estão identificados nem disponíveis para diagnóstico todos os alergénios moleculares potencialmente relevantes e faltam recomendações mais detalhadas sobre a forma como devem ser valorizados na prática clínica, o que limita, atualmente, uma implementação ainda mais alargada. A positividade dos TCP ou slgE/mlgE reflete a existência de sensibilização a determinado alergénio. A sensibilização constitui a fase em que, após contacto com alergénios, e apenas em indivíduos com predisposição para tal, são produzidos anticorpos específicos da subclasse $\operatorname{lgE}$ para determinado alergénio. No entanto, a existência de sensibilização nem sempre se traduz na ocorrência de uma resposta alérgica associada à exposição aos alergénios identificados ${ }^{33}$. De facto, apesar de a presença de sensibilização alergénica ser um fator de risco para o aparecimento de sintomas de rinite, muitos indivíduos sensibilizados a um ou mais alergénios não desenvolvem sintomas de alergia associados a esses alergénios específicos $^{34,35}$. Por outro lado, a negatividade dos TCP ou slgE/mlgE também não exclui sensibilização (nomeadamente local) a determinado alergénio. Assim, o diagnóstico deve ser feito através da correlação entre a história clínica, o exame objetivo, os testes cutâneos/s/gE $\mathrm{E}^{28,30} \mathrm{e}$, eventualmente, complementado com outros exames de diagnóstico.

Outros testes que podem, também, ser realizados para melhor caraterização do diagnóstico, embora generalizadamente menos disponíveis, sobretudo devido ao equipamento e/ou ao tempo de execução necessários, incluem ${ }^{28,30,36}$ :

\section{- Medição de óxido nítrico nasal (nNO) - O óxi-} do nítrico é produzido continuamente nos seios perinasais. Na RA pode estar aumentado na presença de inflamação, ou diminuído se houver obstrução dos seios perinasais por edema ou secreções que impeçam a drenagem. Por este motivo não é o método ideal, podendo eventualmente ter maior valor diagnóstico com medições seriadas para avaliação da resposta de provas de provocação nasal ou noutras patologias nasais, sobretudo ciliares. 
- Rinomanometria / rinometria acústica - Permitem a avaliação da permeabilidade nasal, com medição de fluxo e pressão nasais (rinomanometria), ou das dimensões da cavidade nasal (rinometria acústica).

- Testes de provocação nasal - A provocação nasal direta com os alergénios/estímulos identificados na história clínica é sobretudo útil para a melhor caraterização de doentes polissensibilizados, na rinite ocupacional e alérgica local e na identificação dos alergénios mais relevantes para imunoterapia específica, com possível identificação e medição no fluido nasal dos mediadores químicos e celulares envolvidos, embora seja algo ainda em fase de investigação.

- Testes de provocação conjuntival - No caso de estar associada sintomatologia compatível com rinoconjuntivite.

A importância do adequado diagnóstico da RA está igualmente relacionada com o processo inflamatório comum que afeta as vias respiratórias superiores e inferiores, provavelmente evolutivo, sendo sustentado e amplificado por mecanismos interligados ${ }^{37,38}$. De facto, a rinite alérgica associa-se a diversas comorbilidades (Quadro 2) que provocam um aumento da morbilidade subjacente, salientando-se, pela sua prevalência e relevância clínica, a asma brônquica'. O possível diagnóstico concomitante de asma brônquica deverá ser avaliado
Quadro 2. Comorbilidades associadas à rinite alérgica (Adaptado de ARIA')

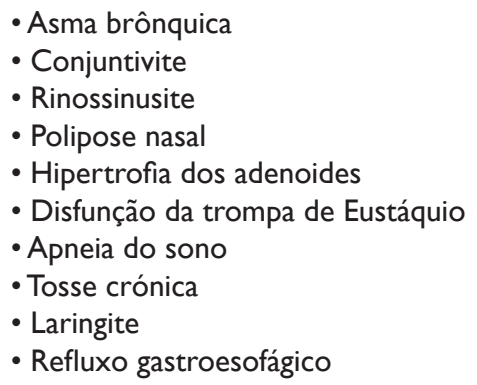

- Asma brônquica

- Conjuntivite

- Rinossinusite

- Polipose nasal

- Hipertrofia dos adenoides

- Disfunção da trompa de Eustáquio

- Apneia do sono

- Tosse crónica

- Laringite

- Refluxo gastroesofágico

através da realização de provas funcionais respiratórias sempre que exista suspeita de envolvimento brônquico'.

\section{Diagnóstico diferencial}

O diagnóstico diferencial inclui alterações estruturais/ /funcionais (sobretudo na presença de obstrução unilateral e/ou síndrome de apneia do sono), doenças sistémicas (sobretudo na presença de infeções respiratórias de repetição ou de outros achados ao exame objetivo/avaliação analítica sugestivos de patologia sistémica) ${ }^{30}$ e outros fenótipos de rinite ${ }^{39}$.

O Quadro 3 sumariza as principais patologias que fazem diagnóstico diferencial com a rinite alérgica. De entre os diagnósticos diferenciais, importa referir, pela proximidade em termos de mecanismos imunológicos e de tratamento, a rinite alérgica local, que é caraterizada

Quadro 3. Diagnóstico diferencial da rinite alérgica. (Adaptado Papadopoulos NG et al ${ }^{23}$.)

\begin{tabular}{|l|l|l|}
\hline Alterações estruturais/funcionais & \multicolumn{1}{|c|}{ Doença sistémica } & \multicolumn{1}{|c|}{ Outros fenótipos de rinite } \\
\hline Desvio do septo nasal & Discinesia ciliar primária & Rinite alérgica local \\
Hipertrofia dos cornetos nasais & Fibrose cística & Rinossinusite crónica \\
Neoplasia da nasofaringe & Síndrome de Churg-Strauss & Rinite infeciosa \\
Hipertrofia dos adenoides & Granulomatose de Wegener & Rinite não alérgica \\
Apneia do sono & Sarcoidose & Rinite mista \\
Refluxo gastroesofágico & Amiloidose & \\
Pólipos nasais & & \\
Atresia das coanas & & \\
Corpo estranho & & \\
Patologia da válvula nasal & & \\
\hline
\end{tabular}


por uma resposta alérgica localizada à mucosa nasal em doentes com TCP e slgE/mlgE negativos. O seu diagnóstico implica a identificação de sensibilização local a nível nasal e é feito através de provas de provocação nasal positivas e/ou a deteção de slgE nasal ${ }^{30}$. Está descrita a possibilidade de coexistência, no mesmo doente, de RA e da rinite alérgica local (dual allergic rhinitis), não devendo a confirmação do diagnóstico de RA excluir automaticamente a possibilidade de existência de sensibilização apenas local a outros alergénios ${ }^{40}$.

\section{Tratamento}

O objetivo primário do tratamento da RA é restaurar a função da via aérea superior, de forma a melhorar a qualidade de vida do doente, nomeadamente no que concerne à sua vida social, sono, olfato e paladar ${ }^{20,24,30}$. Adicionalmente, o controlo atempado da RA poderá permitir evitar a progressão da doença alérgica e a associação a outras patologias ${ }^{41,42}$.
Embora pouca evidência científica o comprove, a evicção dos alergénios envolvidos pode auxiliar no controlo dos sintomas. No entanto, pode ser uma medida de implementação bastante difícil ou praticamente impossível, por exemplo, no caso dos pólenes na época polínica ou no caso dos ácaros do pó doméstico e epitélio de animais, dado serem alergénios ubiquitários e perenes ${ }^{1,20,30}$.

Relativamente ao tratamento farmacológico, existem vários grupos terapêuticos disponíveis que poderão ser selecionados de acordo com o tipo de sintomas predominantes, com a gravidade das queixas e com a preferência do doente (Quadro 4) ${ }^{43}$. Não está contraindicada a utilização de vários grupos terapêuticos em conjunto numa rinite alérgica moderada-grave mal controlada $20,21,27,30$.

Assim:

- Anti-histamínicos HI orais: são eficazes no tratamento da rinorreia, esternutos, prurido nasal e ocular. Os anti-histamínicos de segunda geração

Quadro 4. Tratamento da rinite alérgica (Adaptado de SPAIC ${ }^{43}$ )

\begin{tabular}{|c|c|c|c|c|c|}
\hline & Esternutos & Rinorreia & $\begin{array}{c}\text { Obstrução } \\
\text { nasal }\end{array}$ & Prurido nasal & $\begin{array}{c}\text { Sintomas } \\
\text { oculares }\end{array}$ \\
\hline \multicolumn{6}{|l|}{ Anti-histamínicos $\mathrm{HI}$} \\
\hline Orais & +++ & +++ & $0 /+$ & +++ & ++ \\
\hline Intranasais & ++ & +++ & + & ++ & 0 \\
\hline Intraoculares & 0 & 0 & 0 & 0 & +++ \\
\hline Glucocorticoides intranasais & +++ & +++ & ++ & ++ & + \\
\hline \multicolumn{6}{|l|}{ Cromonas } \\
\hline Intranasais & + & + & + & + & 0 \\
\hline Intraoculares & 0 & 0 & 0 & 0 & ++ \\
\hline \multicolumn{6}{|l|}{ Descongestionantes } \\
\hline Intranasais & 0 & 0 & ++ & 0 & 0 \\
\hline Orais & 0 & 0 & + & 0 & 0 \\
\hline Anticolinérgicos intranasais & 0 & +++ & 0 & 0 & 0 \\
\hline Antileucotrienos orais & + & ++ & ++ & $?$ & ++ \\
\hline
\end{tabular}

Eficácia: 0 - nula; + - ligeira; ++ - moderada; +++ - elevada; ? - desconhecida 
estão recomendados e devem ser usados em detrimento dos de primeira geração, uma vez que têm a vantagem de menor passagem através da barreira hematoencefálica e, consequentemente, menos efeitos secundários (como a sedação) ${ }^{28,44}$.

- Anti-histamínicos $\mathrm{HI}$ tópicos nasais: Está comprovado que reduzem a rinorreia e o prurido nasal, podendo ser utilizados nos doentes que não respondem aos anti-histamínicos $\mathrm{HI}$ orais. Está também descrita a utilização benéfica e sinérgica da associação de anti-histamínico $\mathrm{HI}$ intranasal com glucocorticoide intranasal no tratamento de doentes com rinite moderada-grave $e^{1,45,46}$. De facto, a eficácia e maior rapidez de ação desta associação (vs glucocorticoide intranasal isolado) foi demonstrada em diversos ensaios clínicos randomizados e controlados com placebo, sendo ela atualmente recomendada nas guidelines ARIA como terapêutica de primeira linha na rinite alérgica moderada-grave ${ }^{45,47,48}$. Uma associação de anti-histamínico $\mathrm{HI}$ com glucocorticoide intranasal em dispositivo único ficou disponível em Portugal muito recentemente.

- Glucocorticoides tópicos nasais: São considerados a base do tratamento da RA, sobretudo pela sua ação anti-inflamatória, permitindo o controlo dos quatro principais sintomas da RA: esternutos, prurido, rinorreia e obstrução nasal, com efeito sinérgico se associados ao anti-histamínico $\mathrm{HI}$ oral ${ }^{49}$ ou nasal ${ }^{45,47,50}$. Estão recomendados nas orientações do ARIA como tratamento de eleição da RA, quer nos adultos, quer nas crianças. Não têm absorção sistémica significativa, pelo que apresentam poucos efeitos secundários, sendo os mais frequentes a nível local: epistaxes, cefaleia, faringite, disgeusia ${ }^{27,30}$.

- Anticolinérgicos tópicos nasais: São eficazes no controlo da rinorreia. No entanto, esse é o único sintoma com significativa redução, pelo que estão indicados apenas como tratamento adjuvante quando não é possível o controlo da rinorreia com anti-histamínicos $\mathrm{HI}$ e/ou glucocorticoides tópicos nasais ${ }^{30}$.

- Antagonistas dos leucotrienos: Os leucotrienos são potentes mediadores na asma brônquica e contribuem também para as reações inflamatórias agudas e tardias que ocorrem na RA. Desta forma, os sintomas da RA podem ser amenizados através do uso de fármacos com efeito inibidor dos leucotrienos. Atualmente, apenas os antagonistas do recetor CysLTI são recomendados como terapêutica complementar da RA, sendo o montelucaste o mais bem estudado, sobretudo na rinite persistente moderada-grave ${ }^{1,30}$. O seu possível efeito sinérgico com os anti-histamínicos e/ou glucocorticoides tópicos nasais é, ainda, controverso ${ }^{51}$.

- Cromonas: Funcionam como estabilizadores tópicos dos mastócitos, sendo mais eficazes no controlo dos sintomas oculares do que dos nasais. Tendo em consideração a eficácia inferior relativamente aos anti-histamínicos e glucocorticoides tópicos nasais, bem como a posologia tetradiária, as cromonas são raramente utilizadas como tratamento de manutenção da RA ${ }^{30}$.

- Imunoterapia específica com alergénios: É a única opção terapêutica que modifica o mecanismo imunológico subjacente à RA, induzindo a dessensibilização e produzindo um estado de anergia para os alergénios agressores. Está indicada no tratamento da RA moderada-grave e geralmente é recomendada apenas a partir dos 5 anos de idade, devendo ser administrada com extratos alergénicos padronizados. Vários estudos demonstraram a persistência do efeito da imunoterapia com alergénios nos sintomas nasais e oculares mesmo após a descontinuação do tratamento, tendo vantagens farmacoeconómicas relativamente às outras formas de tratamento da RA ${ }^{1,30}$. Poderá ter ainda um papel protetor no desenvolvimento de asma brônquica e de novas sensibilizações ${ }^{30}$.

- Anticorpo anti-IgE: O omalizumab é um anticorpo monoclonal humanizado recombinante anti- 
-lgE, que interfere na ligação da lgE aos mastócitos, basófilos e eosinófilos. Uma revisão sistemática com meta-análise publicada recentemente mostrou que o omalizumab melhora os sintomas nasais e oculares, reduz o uso de medicação de alívio e melhora a qualidade de vida de doentes com rinite alérgica não controlada ${ }^{52}$. É de referir também que alguns estudos têm mostrado vantagens na sua utilização como pré e cotratamento no início de imunoterapia com alergénios, com aumento da sua eficácia e segurança, podendo ser uma alternativa terapêutica, especialmente em doentes excecionalmente reativos à imunoterapia ${ }^{53-55}$. Considerando os custos elevados do omalizumab, quando comparado com outros medicamentos disponíveis para o tratamento da rinite alérgica, esta estratégia, combinando um tratamento não modificador da evolução da doença (como é o omalizumab) com um tratamento que modifica o mecanismo imunológico subjacente à doença alérgica (como é a imunoterapia com alergénios), aplicada a doentes selecionados, poderia melhorar a relação custo-benefício da sua utilização nesta patologia ${ }^{52}$. No entanto, não existem atualmente estudos que demonstrem o custo-eficácia da utilização do omalizumab em doentes com rinite alérgica, incluindo como adjuvante no início de imunoterapia com alergénios.

- Descongestionantes nasais: Têm grande eficácia no controlo da congestão nasal, mas pelos seus efeitos secundários, nomeadamente o efeito rebound e a associação com o desenvolvimento de rinite medicamentosa, devem ser utilizados por um período máximo de 3 a 5 dias $^{30}$.

- Glucocorticoides orais: Podem ser utilizados por períodos curtos, estando indicados em fase de agudização quando as outras opções terapêuticas não obtiveram controlo sintomático. $O$ seu uso deve ser parcimonioso, sobretudo pelos seus efeitos secundários ${ }^{30}$.
- Lavagens nasais com solução salina: A lavagem nasal é uma terapêutica não farmacológica complementar que permite alívio instantâneo e momentâneo dos sintomas de RA, sobretudo a obstrução e o prurido nasais ${ }^{30}$.

- Anti-histamínicos tópicos oculares: Podem ser úteis nas situações em que há conjuntivite alérgica associada $^{30}$.

\section{CONCLUSÕES E RECOMENDAÇÕES}

O doente descrito na vinheta clínica apresenta um quadro clínico compatível com rinoconjuntivite alérgica moderada-grave, persistente, polissensibilizado a ácaros do pó doméstico e pólenes. A resposta ao tratamento com glucocorticoide tópico nasal e anti-histamínico $\mathrm{HI}$ oral e tópico ocular foi apenas parcial, pelo que se ponderou iniciar imunoterapia específica. Realizou teste molecular ImmunoCAP ISAC ${ }^{\circledR}$ -ThermoFisher Scientific, Sweden, com positividade para os componentes moleculares de: I) pólen de gramíneas - nCyn dI (I,8 ISU-E), rPhl pI (8,5 ISU-E), rPhl pII (4,2 ISU-E); 2) pólen de ervas - nSal kI (I2 ISU-E); e 3) ácaros do pó doméstico - nDer fl (3,5 ISU-E), rDer f2 (4,9 ISU-E), nDer pI (7,4 ISU-E) e rDer p2 (8,6 ISU-E). Tendo em conta o perfil de sensibilização obtido e o agravamento dos sintomas na primavera e outono, o doente iniciou imunoterapia específica subcutânea, em pauta rush, com polimerizado de $50 \%$ Dermatophagoides pteronyssinus e 50\% gramíneas (Clustoid, Roxall ${ }^{\circledR}$ ). Cerca de dois anos depois, o doente refere redução gradual dos sintomas, necessitando de medicação apenas por períodos durante a primavera. A rinite alérgica é muito prevalente, mas frequentemente subdiagnosticada e subtratada, apesar do forte impacto que tem na qualidade de vida, capacidade de trabalho e rendimento escolar. Este caso clínico reflete a importância da aplicação de uma medicina de precisão nos doentes com rinite alérgica através do diagnóstico e posterior tratamento personalizados. 


\section{Conflito de interesses}

Os autores declaram que não existem conflitos de interesses.

\section{ORCID:}

Leonor Esteves Caldeira (D) 0000-0003-2347-4658

Maria Inês T. Silva (iD) 0000-0002-804I-1306

Gonçalo Martins-dos-Santos (D) 0000-0002-5726-5486

Ana Margarida Pereira (D) 0000-0002-5468-0932

Contactos:

Leonor Esteves Caldeira

Serviço de Imunoalergologia, Hospital Santa Maria

Avenida Professor Egas Moniz

1649-035, Lisboa

Email: leonorfsecaldeira@gmail.com

Maria Inês T. Silva

Serviço de Imunoalergologia, Hospital Santa Maria

Avenida Professor Egas Moniz

1649-035, Lisboa

Email: inescerca92@gmail.com

\section{REFERÊNCIAS}

I. Bousquet J, Khaltaev N, Cruz AA, Denburg J, Fokkens WJ, Togias $A$, et al. Allergic rhinitis and its impact on asthma (ARIA) 2008*: ARIA: 2008 Update. Allergy 2008;63(68):8-160.

2. Katelaris CH, Lee BW, Potter PC, Maspero JF, Cingi C, Lopatin $A$, et al. Prevalence and diversity of allergic rhinitis in regions of the world beyond Europe and North America. Clin Exp Allergy 20I2;42(2):186-207.

3. Morais-Almeida M, Pite H, Pereira AM, Todo-Bom A, Nunes C, Bousquet J, et al. Prevalence and classification of rhinitis in the elderly: a nationwide survey in Portugal. Allergy 2013;68: II50-7.

4. Bauchau V, Durham SR. Prevalence and rate of diagnosis of allergic rhinitis in Europe. Eur Respir J 2004;24: 758-64.

5. Morais-Almeida M, Santos N, Pereira AM, Branco-Ferreira M, Nunes C, Bousquet J, et al. Prevalence and classification of rhinitis in preschool children in Portugal: a nationwide study. Allergy 2013;68: 1278-88.

6. Gani F, Lombardi C, Barrocu L, Landi M, Ridolo E, Bugiani M, et al. The control of allergic rhinitis in real life: a multicenter crosssectional Italian study. Clin Mol Allergy 2018;16: 4.

7. Nolte H, Nepper-Christensen S, Backer V. Unawareness and undertreatment of asthma and allergic rhinitis in a general population. Respir Med 2006;100: 354-62.

8. Canonica GW, Bousquet J, Mullol J, Scadding GK, Virchow JC. A survey of the burden of allergic rhinitis in Europe. Allergy 2007;62 Suppl 85:17-25.
9, Bousquet J, Bullinger M, Fayol C, Marquis P, Valentin B, Burtin B. Assessment of quality of life in patients with perennial allergic rhinitis with the French version of the SF-36 Health Status Questionnaire. J Allergy Clin Immunol 1994;94: 182-8.

10. Meltzer EO, Nathan RA, Seiner JC, Storms W. Quality of life and rhinitic symptoms: Results of a nationwide survey with the SF-36 and RQLQ questionnaires.J Allergy Clin Immunol 1997;99: S8I5-S8I9.

II. Meltzer EO. Quality of life in adults and children with allergic rhinitis. J Allergy Clin Immunol 200I;108: S45-53.

12. Roger A, Arcalá Campillo E, Torres MC, Millan C, Jáuregui I, Mohedano E, et al. Reduced work/academic performance and quality of life in patients with allergic rhinitis and impact of allergen immunotherapy. Allergy Asthma Clin Immunol 2016;12:40.

13. Bédard A, Antó JM, Fonseca JA, Arnavielhe S, Bachert C, Bedbrook $A$, et al. Correlation between work impairment, scores of rhinitis severity and asthma using the MASK-air ${ }^{\circledR}$ App. Allergy 2020;75:1672-88.

14. Bousquet J, Bewick M, Arnavielhe S, Mathieu-Dupas E, Murray $\mathrm{R}$, Bedbrook $\mathrm{A}$, et al. Work productivity in rhinitis using cell phones: The MASK pilot study. Allergy 2017;72(10):1475-84.

15. Vandenplas O, Vinnikov D, Blanc PD, Agache I, Bachert C, Bewick $M$, et al. Impact of rhinitis on work productivity: A systematic review. J Allergy Clin Immunol Pract 2018;6(4):1274-86.e9.

16. Avdeeva KS, Reitsma S, Fokkens WJ. Direct and indirect costs of allergic and non-allergic rhinitis in the Netherlands. Allergy 2020.

17. Colás C, Brosa M, Antón E, Montoro J, Navarro A, Dordal MT, et al. Estimate of the total costs of allergic rhinitis in specialized care based on real-world data: the FERIN Study. Allergy 2017;72: 959-66.

18. Eifan AO, Durham SR. Pathogenesis of rhinitis. Clin Exp Allergy 2016;46(9):1139-1151.

19. Sin B, Togias A. Pathophysiology of allergic and nonallergic rhinitis. Proceedings of the American Thoracic Society 201 I;8(I):106-14.

20. Min Y-G. The pathophysiology, diagnosis and treatment of allergic rhinitis. Allergy Asthma Immunol Res 2010;2(2):65-76. doi:10.4168/aair.2010.2.2.65

21. Bjermer L, Westman M, Holmström M, Wickman MC. The complex pathophysiology of allergic rhinitis: scientific rationale for the development of an alternative treatment option. Allergy Asthma Clin Immunol 2019;15(24):24.

22. Hansen I, Klimek L, Mösges R, Hörmann K. Mediators of inflammation in the early and the late phase of allergic rhinitis. Curr Opin Allergy Clin Immunol 2004;4(3):159-63.

23. Papadopoulos NG, Bernstein JA, Demoly P, Dykewicz M, Fokkens $W$, Hellings PW et al. Phenotypes and endotypes of rhinitis and their impact on management: a PRACTALL report. Allergy 20I5;70(5):474-494.

24. Wallace DV, Dykewicz MS, Bernstein DI, Blessing-Moore J, Cox L, Khan DA, et al. The diagnosis and management of rhinitis: An updated practice parameter. J Allergy Clin Immunol 2008;I22(2):SI-S84. 


\section{RINITE ALÉRGICA - CLASSIFICAÇÃO, FISIOPATOLOGIA, DIAGNÓSTICO E TRATAMENTO / PÁGINA EDUCACIONAL - ARTIGO DE REVISÃO}

25. Ng MLS, Warlow RS, Chrishanthan N, Ellis C, Walls R. Preliminary criteria for the definition of allergic rhinitis: a systematic evaluation of clinical parameters in a disease cohort (I). Clin Exp Allergy 2000;30:1314-31.

26. Ng MLS, Warlow RS, Chrishanthan N, Ellis C, Walls RS. Preliminary criteria for the definition of allergic rhinitis: a systematic evaluation of clinical parameters in a disease cohort (II). Clin Exp Allergy 2000;30:1417-22.

27. Brożek JL, Bousquet J, Agache I, Agarwal A, Bachert C, Bosnic-Anticevich S, et al. Allergic Rhinitis and its Impact on Asthma (ARIA) guidelines - 2016 revision. J Allergy Clin Immunol 20I7;140(4):950-8.

28. Simons FER. HI Antihistamines: Current status and future directions. World Allergy Organ J 2008:145-55.

29. Matricardi PM, Dramburg S, Potapova E, Skevaki C, Renz H. Molecular diagnosis for allergen immunotherapy. J Allergy Clin Immunol 2019;143(3):831-43.

30. Akdis CA, Hellings PW, Agache I. Global atlas of allergic rhinitis and chronic rhinosinusitis; European Academy of Allergy and Clinical Immunology (EAACI), 20I5; 150-164; 187-207; 222-5.

31. Brunetto B, Tinghino R, Braschi MC, Antonicelli L, Pini C, lacovacci P. Characterization and comparison of commercially available mite extracts for in vivo diagnosis. Allergy 2010; 65: 184-90.

32. Matricardi PM, Kleine-Tebbe J, Hoffmann HJ, Valenta R, Hilger $C$, Hofmaier $\mathrm{S}$ et al. EAACI molecular allergology user's guide. Pediatr Allergy Immunol 2016; 27(suppl 23):I-250.

33. Ansotegui IJ, Melioli G, Canoninca GW, Caraballo L, Villa E, Ebisawa $M$ et al. IgE allergy diagnostics and other relevant tests in allergy, a World Allergy Organization position paper. World Allergy Organ J 2020;13:100080.

34. Settipane RJ, Hagy GW, Settipane GA. Long-term risk factors for developing asthma and allergic rhinitis: a 23-year follow-up study of college students. Allergy Proc 1994;15(I):2I-5.

35. Droste JH, Kerhof M, de Monchy JG, Schouten JP, Rijcken B. Association of skin test reactivity, specific lgE, total IgE, and eosinophils with nasal symptoms in a community-based population study. The Dutch ECRHS Group. J Allergy Clin Immunol 1996;97(4):922-32.

36. Skoner DP. Allergic rhinitis: Definition, epidemiology, pathophysiology, detection, and diagnosis. J Allergy Clin Immunol 200I;108(I):S2-S8.

37. Compalati E, Ridolo E, Passalacqua G, Braido F, Villa E, Canonica GW. The link between allergic rhinitis and asthma: the united airways disease. Expert Rev Clin Immunol 2010;6(3):413-23.

38. Shaaban R, Zureik M, Soussan D, Neukirch C, Heinrich J, Sunyer J, et al. Rhinitis and onset of asthma: a longitudinal populationbased study. Lancet 2008; 372: 1049-57.

39. Wheatley L, Togias A. Allergic rhinitis. N Engl J Med. 20I5;29;372(5):456-63. doi:I0.1056/NEJMcpI4I2282
40. Eguiluz-Gracia I, Fernandez-Santamaria R, Testera-Montes A, Ariza A, Campo P, Prieto A et al. Coexistence of nasal reactivity to allergens with and without IgE sensitization in patients with allergic rhinitis. Allergy 2020;75:1689-98.

4I. Lin J, Gao J, Lai K, Zhou X, He B, Zhou J et al. The characteristic of asthma control among nasal diseases population: Results from a cross-sectional study. Plos One 13(2):e0191543.

42. Oka A, Hirano T, Yamaji Y, Ito K, Oishi K, Edakuni N et al. Determinants of incomplete asthma control in patients with allergic rhinitis and asthma. J Allergy Clin Immunol Pract 2017;5(I):160-4.

43. Pereira AM, Gonçalves C, Ferreira J, Costa RP. Folheto Rinite, SPAIC, https://www.spaic.pt/client_files/files/Folheto\%20Rinite. pdf, consultado a 27/0I/2020.

44. Bousquet J, Schünemann HJ, Samolinski B, Demoly P, Baena-Cagnani $C E$, Bachertet $C$, et al. Allergic Rhinitis and its Impact on Asthma (ARIA): Achievements in 10 years and future needs. J Allergy Clin Immunol 2012;130(5):1049-62.

45. Bousquet J, Schünemann H, Togias A, Bachert C, Erhola M, Hellings PW, et al. Next-generation allergic rhinitis and its impact on asthma (ARIA) guidelines for allergic rhinitis based on Grading of Recommendations Assessment, Development and Evaluation (GRADE) and real-world evidence. J Allergy Clin Immunol 2020; I45(I):70.80.

46. Ratner P, Hampel F, Van Bavel J, Amar NJ, Daftary P, Wheeler $\mathrm{W}$, et al. Combination therapy with azelastine hydrochloride nasal spray and fluticasone propionate nasal spray in the treatment of patients with seasonal allergic rhinitis. Ann Allergy Asthma Immunol 2008;100(I):74-8I

47. Carr W, Bernstein J, Lieberman P, Meltzer E, Bachert C, Price $D$, et al. A novel intranasal therapy of azelastine with fluticasone for the treatment of allergic rhinitis. J Allergy Clin Immunol 2012;129(5):1282-9.

48. Meltzer E, Ratner P, Bachert C, Carr W, Berger W, Canonica $\mathrm{GW}$ et al. Clinically relevant effect of a new intranasal therapy (MP29-02) in allergic rhinitis assessed by responder analysis. Int Arch Allergy Immunol 2013;16I(4):369-77.

49. Bousquet J, Hellings PW, Aberer W, Agache I, Akdis CA, Akdis $M$, et al. Next-generation ARIA care pathways for rhinitis and asthma: a model for multimorbid chronic diseases. Clin Transl Allergy 2019;9(44).

50. Haahr PA, Jacobsen C, Christensen ME. MP-AzeFlu provides rapid and effective allergic rhinitis control: results of a non-interventional study in Denmark. Int Forum Allergy Rhinol 2019;00:I-8.

5I. Lavorini F, Matucci A, Rossi O, Pistolesi M. Concomitant bilastine and montelukast as additive therapy for seasonal allergic rhinoconjunctivits and mild to moderate asthma. The SKY study. Allergy 2020;75(3):675-7. doi: 10.1III/all.14007

52. Yu C, Wang K, Cui X, Lu L, Dong J, Wang M et al. Clinical efficacy and safety of omalizumab in the treatment of allergic rhinitis: A systematic review and meta-analysis of randomized clinical 
trials. Am J Rhinol Allergy 2020;34(2):196-208. doi: 10.1177/ 1945892419884774

53. Casale TB, Busse WW, Kline JN, Ballas ZK, Moss MH, Townley $R G$ et al. Omalizumab pretreatment decreases acute reactions after rush immunotherapy for ragweed-induced seasonal allergic rhinitis. J Allergy Clin Immunol 2006; I 17(I):134-40.

54. Kamin W, Kopp MV, Erdnuess F, Schauer U, Zielen S, Wahn $U$. Safety of anti-lgE treatment with omalizumab in children with seasonal allergic rhinitis undergoing specific immunotherapy simultaneously. Pediatr Allergy Immunol 20I0;2I (I Pt 2):el60-5.

55. Kopp MV, Hamelmann E, Zielen S, Kamin W, Bergmann KC, Sieder $\mathrm{C}$ et al. Combination of omalizumab and specific immunotherapy is superior to immunotherapy in patients with seasonal allergic rhinoconjunctivitis and co-morbid seasonal allergic asthma. Clin Exp Allergy 2009;39(2):27I-9. 